\title{
Emotional Delivery in Pro-social Crowdfunding Success
}

\author{
Lauren Rhue \\ Wake Forest University \\ School of Business \\ Winston-Salem, NC 27106, USA \\ rhuela@wfu.edu
}

Lionel P. Robert Jr.

University of Michigan

School of Information

Ann Arbor, MI 48109, USA

Iprobert@umich.edu

Permission to make digital or hard copies of part or all of this work for personal or classroom use is granted without fee provided that copies are not made or distributed for profit or commercial advantage and that copies bear this notice and the full citation on the first page. Copyrights for third-party components of this work must be honored. For all other uses, contact the Owner/Author.

CHI'18 Extended Abstracts, April 21-26, 2018, Montreal, QC, Canada (c) 2018 Copyright is held by the owner/author(s).

-5621-3/18/04.

https://doi.org/10.1145/3170427.3188534

\begin{abstract}
How do fundraisers effectively use emotions in prosocial crowdfunding platforms? Pro-social crowdfunding platforms allow for online campaigns to raise money, and these fundraisers rely more heavily on emotions to attract backers because there are no financial incentives to contribute to these campaigns. People give to pro-social crowdfunding campaigns out of empathy and sympathy for the campaign beneficiaries. Using prior research on empathy, we considered the emotional drivers of empathy and examined how campaigns convey those emotions using campaign pictures and language. We analyzed the facial expressions in campaign pictures and the emotional words in the campaign language, and we found that emotions shape fundraising success. Furthermore, our study shows that the same emotion - happiness/joy leads to different fundraising success based on whether it is conveyed visually or textually. A future experiment will more explicitly connect these emotions to empathy in potential backers.
\end{abstract}

\section{Author Keywords}

Crowdfunding; emotions; facial recognition; text analysis; e-commerce; online markets; empathy. 


\section{GoFundMe}

Purpose: GoFundMe users can start a fundraising campaign for any reason for themselves or for others. This beneficiary option makes GoFundMe unique among the most popular crowdfunding options.

Campaigns: Each campaign profile contains standard information about the campaign and fundraiser.

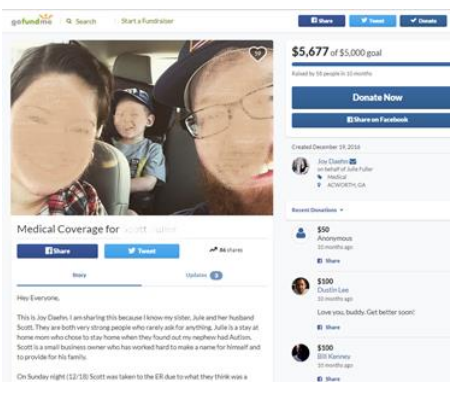

Figure 1. A sample campaign on GoFundMe. Each campaign contains a picture, a title, a text description, the target fundraising goal, the amount of money raised, and the number of campaign contributors.

\section{ACM Classification Keywords}

H.5.m. Information interfaces and presentation (e.g., $\mathrm{HCI}$ ): Miscellaneous.

\section{Introduction}

Crowdfunding campaigns are becoming an increasingly popular funding mechanism. In 2016, $22 \%$ of U.S. adults contributed to some type of a crowdfunding campaign [17]. In general, crowdfunding platforms allow people "to fund their ventures by drawing on relatively small contributions from a relatively large number of individuals using the internet" [12]. Charitable or donation-based crowdfunding platforms allow fundraisers to solicit money without offering financial incentives.

Crowdfunding platforms enable fundraisers to share "soft information" with potential backers via the campaign picture and text description [4], and we know that the availability of these media influences success [9]. Yet many questions remain with regard to the role of emotions in facilitating fundraising [8]. For example, does the type of emotion (e.g., happy vs. sad) attract more backers, or more money? Does it matter whether emotions are displayed by text or by photos?

The goal of this study is to identify the emotional elements conveyed through text and facial expressions that are likely to encourage donors. To accomplish this, we employed novel image and text analysis techniques to examine the impact of emotions on the success of 32,000 charitable campaigns. In doing so, we sought to contribute to the literature on crowdfunding by focusing on (1) charitable campaigns and (2) the role of emotion displayed by both language and facial expressions. Our paper contributes to the literature by showing that emotions in images and language are critical elements in fundraising success on charitable platforms.

\section{Theory Background}

Literature on crowdfunding has identified several

factors that are important on crowdfunding platforms.

These factors include number of updates, goal size, and whether a project is featured [12]; herding and geographic proximity [2]; demographics [15, 19]; and textual phrases [11]. This literature has also identified the importance of topics [18], sentiment [3], and the presence of pictures (and emotions derived from profile pictures) $[5,8]$.

We focused on emotion because prior literature has shown that emotion is particularly important for charitable giving $[1,16]$. Emotions have been shown to drive offline [10] and online philanthropic giving [6].

Emotions are particularly important here because these campaigns provide no financial benefit.

This study builds on the literature focusing on the role of emotions through facial expressions. Generally, facial expressions have been used as a signal of creditability to engender trust in rewards-based crowdfunding platforms like Kickstarter [8]. We extend this research by examining text as well as the emotional signals generated in the pictures.

\section{Data}

This study uses data from GoFundMe, a crowdfunding platform for users to broadcast open-ended monetary requests to others. More details on GoFundMe are available in the inset (Figure 1). 


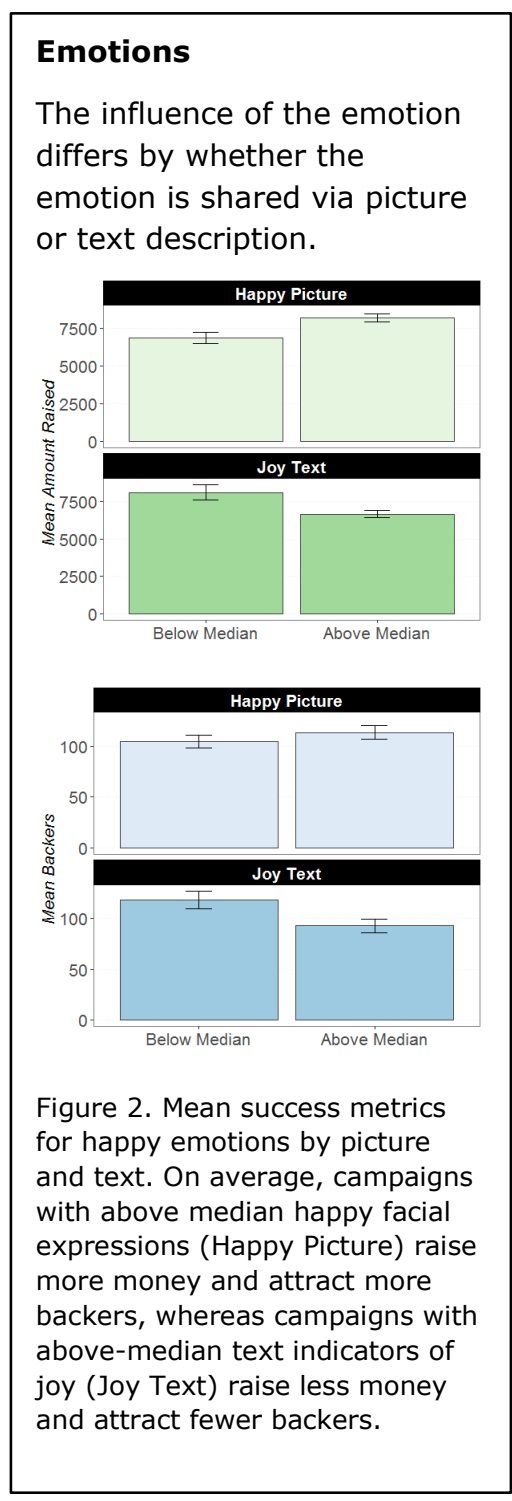

To gather GoFundMe data, we scraped campaigns from each of the categories and subcategories from the homepage. All of the campaigns were accessible from the GoFundMe homepage, and we captured their positions in the homepage. We restricted the data to campaigns located in the United States and those raising money in U.S. dollars with a text description, leading to an analysis of more than 32,000 campaigns.

\section{GoFundMe Variables}

Our data set contains two success metrics: the amount of money raised by the campaign and the number of the campaign backers. We also captured a number of control variables for the campaigns: campaign category, the year that the campaign launched, the fundraiser's state, the number of favorites, and the campaign fundraiser's gender (based on the gender of the first name according to the Social Security Administration).

\section{Emotion in Photos}

This study builds on the prior research to catalog emotion from videos [14] and pictures [8]. We used image classification software, Face++, to identify the emotions in the facial expressions of the campaign pictures on a scale of $0-100$. We primarily focused on faces with happy, sad, and neutral expressions, and we classified the continuous emotional index into a binary indicator of above median or below median emotion [3] (Figure 2).

\section{Emotion in Text}

We classified the emotions of the campaign language using the NRC Emotional Lexicon [13]. We classified each word in the description according to the NRC dictionary, and then aggregated the number of words in each emotional category to produce a total number of words in each emotional category (joy, sadness, negativity, and positivity). We normalized the count of emotional words using the total number of words in the description to create an emotion-specific index for the campaign description, normalized by the length of the description. We then split this index into a binary indicator for whether the emotion was above or below the overall median [3] (Figure 2).

\section{Results and Discussion}

We estimated two ordinary least squares (OLS) models, one with the log-transformed count of the backers as the dependent variable and the other with the amount of money raised as the dependent variable. The variables of interest are the binary indicators for the emotions in the photos (happy, sad, and neutral) and the emotion in the text (joy, sadness, negative sentiment, positive sentiment), and we controlled for the campaign characteristics mentioned in the prior section.

Figure 3 shows the coefficient estimates for the variables of interest in the regression of the emotions in the picture and text on the success metrics: the logtransformed count of backers and log-transformed amount of money raised. 


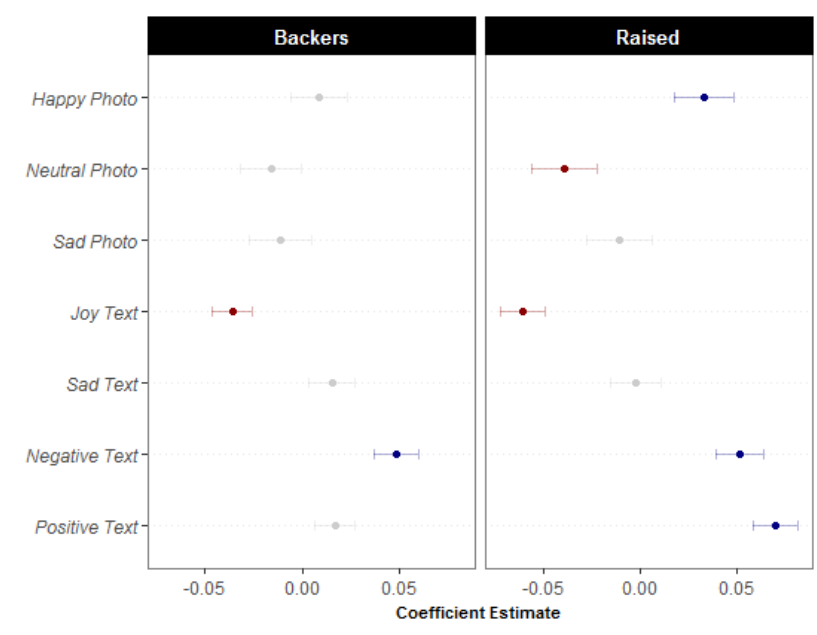

Figure 3. Coefficient estimates for emotions and crowdfunding success. Red and blue indicate significant negative and positive coefficients, respectively, and gray points are not significant.

Our preliminary analysis uncovered a number of key findings. First, the facial expressions in the campaign pictures influence the amount of money raised and the number of the campaign contributors. Campaign photos of people with happy facial expressions raised more money, whereas the campaign photos with neutral facial expressions raised less money. Second, the emotional language in the campaign description influences the amount of money raised and the count of campaign contributors. Campaign descriptions with positive or negative sentiment raised more money, further indicating the importance of the emotional content in pro-social campaigns. Last, joyful language is associated with less money raised. This observation directly contradicts the findings that happy facial expressions lead to increased donations, suggesting that the importance of the emotional content differs based on whether the emotion is conveyed through a photo or through text.

In addition to the emotion-based variables, we analyzed other elements of the campaign picture such as the number of faces and the age of the subjects in the campaign picture. Campaign pictures with more people or with a child increased the number of backers and the amount of money raised. Campaigns written on behalf of another person consistently raised more money and attracted more backers.

The model also included a number of control variables to account for differences across campaigns. We classified the campaigns by their GoFundMe category (e.g., Memorial Funds, Travel, Emergency) and included category-level indicators in the model. We also included time, location, and demographic indicators in all models. Across models, the $\mathrm{R}^{2}$ values ranged $0.594-$ 0.598 .

These findings highlight how visual and language-based emotions generate different responses. Happy facial expressions increase contributions whereas joyful language decreases contributions. We also provide additional evidence of the importance of some emotion in the campaign pictures and campaign descriptions. Campaign pictures with neutral facial expressions or campaigns without positive or negative sentiment received fewer contributions. In summary, our work points to the importance of emotion in crowdfunding campaigns as well as the choice of how to convey that emotion: visually or through language. 


\section{Future Work}

Our next step is to launch an experiment to confirm how the visual and textual emotions translate into empathy for the campaign beneficiary. This experiment will compare how these different conditions generate empathy through emotions and how that translates into donations. The three conditions are:

- Facial expression in the photo: sadness, happiness and neutrality

- Emotional content in the campaign language: sadness and happiness

- Language perspective: first-person and third-person perspectives

Our future studies should enable us to make causal statements about the facial expression and languagebased emotions that generate empathy in the context of individual donations. This is expected to complement our current observational study to help us better understand the role of emotions conveyed through language and facial expressions on the success of charitable crowdfunding.

\section{Conclusion}

Pro-social crowdfunding platforms rely more heavily on the emotions to attract backers because there is no financial incentive to contribute to these campaigns. Our work examines the ways that these campaigns use images and text to capture emotions, and how those emotions translate into fundraising success. Our next study will examine how these emotions generate empathy in potential backers, and how empathy shapes fundraising success.

\section{References}

1. J. Andreoni. 1990. Impure altruism and donations to public goods: A theory of warm-glow giving. The Economics Journal 100 (401), 464-477.

2. A. Agrawal, C. Catalini, and A. Goldfarb. 2015. Crowdfunding: Geography, social networks, and the timing of investment decisions. Journal of Economics \& Management Strategy 24 (2),253274.

3. Tim Althoff, Cristian Danescu-Niculescu-Mizil, and Dan Jurafsky. 2014. How to ask for a favor: A case study on the success of altruistic requests. In Proceedings of ICWSM.

4. P. Belleflamme, N. Omrani, and Martin Peitz. 2015 The economics of crowdfunding platforms. Information Economics and Policy 33 (4), 11-28.

5. J. Duarte and S. Siegel. 2012. Trust and credit: The role of appearance in peer-to-peer lending. The Review of Financial Studies 25 (8), 2455-2483.

6. E. M. Gerber and J. Hui. 2013. Crowdfunding: Motivations and deterrents for participation. ACM Transactions on Computer-Human Interaction (TOCHI) 20 (6), 34.

7. J. T. Hancock, C. Landrigan, and C. Silver. 2007. Expressing Emotion in Text-based Communication. In Proceedings of the SIGCHI Conference on Human Factors in Computing Systems, ACM, New York, NY, 929-932.

8. J. Kim and J. Park. 2017. Does Facial Expression Matter Even Online? An Empirical Analysis of Facial Expression of Emotion and Crowdfunding Success. In Proceedings of the International Conference on Information Systems, Seoul, South Korea.

9. J. Koch and M. Siering. 2015. Crowdfunding Success Factors: The Characteristics of Successfully 
Funded Projects on Crowdfunding Platforms. In Proceedings of the 23rd European Conference on Information Systems (ECIS 2015); Muenster, Germany April 2015. Retrieved from https://ssrn.com/abstract $=2808424$

10. L. Liu, A. Suh, and C. Wagner. 2017. Donation Behavior in Online Micro Charities: An Investigation of Charitable Crowdfunding Projects. In Proceedings of the 50th Hawaii International Conference on System Sciences.

11. T. Mitra and E. Gilbert. 2014. The Language that gets People to Give: Phrases that Predict Success on Kickstarter. In Proceedings of the 17th ACM Conference on Computer-supported Cooperative Work \& Social Computing, ACM, New York, NY, 4961.

12. Ethan Mollick. 2014. The dynamics of crowdfunding: An exploratory study. Journal of Business Venturing 29 (1), 1-16.

13. S. Mohammad and P. Turney. 2013. Crowdsourcing a Word-Emotion Association Lexicon, Computational Intelligence 29 (3), 436-465.

14. J. Reyes and C. Bahm. 2016. Crowdfunding: Applying Collective Indexing of Emotions to Campaign Videos. In Proceedings of the 19th ACM Conference on Computer-supported Cooperative Work \& Social Computing Companion, ACM, New York, NY, 385-388.

15. L. Rhue and J. Clark. 2016. Who Gets Started on Kickstarter? Racial Disparities in Crowdfunding Succes. Working Paper. Available on SSRN at: https://papers.ssrn.com/sol3/papers.cfm?abstract_ id $=2837042$

16. D. Small and N. Verrochi. 2009. The face of need: Facial emotion expression on charity advertisements. Journal of Marketing Research 46 (6), 777-787.

17. A. Smith. 2016. Shared, Collaborative and On Demand: The New Digital Economy. Pew Research.
Retrieved from

http://www.pewinternet.org/2016/05/19/the-newdigital-economy/

18. H. Yuan, R. Y. Lau, and W. Xu. 2016. The determinants of crowdfunding success: A semantic text analytics approach. Decision Support Systems $91,67-76$.

19. P. Younkin and V. Kuppuswamy. 2017. The Colorbind Crowd? Founder Race and Performance in Crowdfunding. Management Science, Articles in Advance. Available online at: https://doi.org/10.1287/mnsc.2017.2774. 